\title{
Correction: Shupeng, W., et al. Carbon Dioxide Retrieval from TanSat Observations and Validation with TCCON Measurements. Remote Sensing 2020, 12(14), 2204
}

\author{
Shupeng Wang ${ }^{1,2}$, Ronald J. van der A ${ }^{2}$, Piet Stammes ${ }^{2}$, Weihe Wang ${ }^{1}$, Peng Zhang ${ }^{1}{ }^{\circledR}$, \\ Naimeng Lu ${ }^{1}$, Xingying Zhang ${ }^{1}$, Yanmeng Bi ${ }^{1}$, Ping Wang ${ }^{2}$ and Li Fang ${ }^{3, *}$ \\ 1 National Satellite Meteorological Center, China Meteorological Administration, Beijing 100081, China; \\ wangsp@cma.cn (S.W.); wangwh@cma.cn (W.W.); zhangp@cma.cn (P.Z.); lunm@cma.cn (N.L.); \\ zxy@cma.cn (X.Z.); biym@cma.cn (Y.B.) \\ 2 Royal Netherlands Meteorological Institute (KNMI), 3731 GA De Bilt, The Netherlands; \\ avander@knmi.nl (R.J.v.d.A.); piet.stammes@knmi.nl (P.S.); ping.wang@knmi.nl (P.W.) \\ 3 Aerospace Information Research Institute, Chinese Academy of Sciences, Beijing 100101, China \\ * Correspondence: fangli@aircas.ac.cn
}

The authors wish to make the following corrections to this paper [1]:

Change in Main Body Paragraphs

1. We have recently been made aware by the TCCON partners that the Beijing site is not currently part of TCCON network. So the authors wish to include more detailed introduction to Beijing site in Section 2.2. The first two sentences of paragraph 2 in Section 2.2 currently reads as follows:

[The validation of retrievals with target mode is conducted using the ground-based FTS measurements in Beijing. The Bruker FTS 125HR in Beijing is similar to the equipment used at TCCON sites and has made observations since 2016 [31].]

To set straight the scientific record we would like to make the following corrections:

[The validation of retrievals with target mode is conducted using the ground-based FTS measurements in Beijing. The Beijing site is not part of the TCCON observation network. The site is operated by National Satellite Meteorological Center, China Meteorological Administration. The Bruker FTS 125HR in Beijing is similar to the equipment used at TCCON sites and has made observations since 2016 [31]. The precision of FTS CO2 column has been reported to be better than $0.2 \%$ under clear sky conditions [31]; however, systemic bias needs be removed through continuous comparison with other FTS of TCCON sites.].

2. We have recently been made aware by the TCCON Network and the Remote Sensing Editorial Offices of improper citation of TCCON data used in our recent paper. The authors wish to include proper reference of each TCCON station used in our study in Section 4.2. The first sentence of said Section 4.2 currently reads as follows:

[In this section, the XCO2 data retrieved with TanSat are validated against the collocated ground-based measurements at TCCON sites.]

To set straight the scientific record we would like to make the following corrections:

[In this section, the XCO2 data retrieved with TanSat are validated against the collocated ground-based measurements at TCCON sites listed in Table 3.] 
Table 3. Geolocation and reference of each TCCON station used in comparison and validation.

\begin{tabular}{ccccc}
\hline Stations & Nationality & Latitude & Longitude & Reference \\
\hline Sodankyla & Finland & $67.3 \mathrm{~N}$ & $26.6 \mathrm{E}$ & {$[44]$} \\
Bialystok & Poland & $53.2 \mathrm{~N}$ & $23.0 \mathrm{E}$ & {$[45]$} \\
Bremen & Germany & $53.1 \mathrm{~N}$ & $8.8 \mathrm{E}$ & {$[46]$} \\
Karlsruhe & Germany & $49.1 \mathrm{~N}$ & $8.4 \mathrm{E}$ & {$[47]$} \\
Park Falls & WI(USA) & $48.4 \mathrm{~N}$ & $2.3 \mathrm{E}$ & {$[48]$} \\
Paris & France & $48.4 \mathrm{~N}$ & $2.3 \mathrm{E}$ & {$[49]$} \\
Izana & Tenerife & $48.4 \mathrm{~N}$ & $2.3 \mathrm{E}$ & {$[50]$} \\
Orleans & France & $47.9 \mathrm{~N}$ & $2.1 \mathrm{E}$ & {$[51]$} \\
Garmisch & Germany & $47.4 \mathrm{~N}$ & $11.0 \mathrm{E}$ & {$[52]$} \\
Rikubetsu & Japan & $43.4 \mathrm{~N}$ & $143.7 \mathrm{E}$ & {$[53]$} \\
Lamont & OK(USA) & $36.6 \mathrm{~N}$ & $97.4 \mathrm{~W}$ & {$[54]$} \\
Tsukuba & Japan & $36.0 \mathrm{~N}$ & $140.1 \mathrm{E}$ & {$[55]$} \\
Saga & Japan & $33.2 \mathrm{~N}$ & $130.2 \mathrm{E}$ & {$[56]$} \\
Darwin & Australia & $12.4 \mathrm{~S}$ & $130.9 \mathrm{E}$ & {$[57]$} \\
Wollongong & Australia & $34.4 \mathrm{~S}$ & $150.8 \mathrm{E}$ & {$[58]$} \\
Burgos & Philippines & $18.5 \mathrm{~N}$ & $120.6 \mathrm{E}$ & {$[59]$} \\
Edwards & USA & $34.9 \mathrm{~N}$ & $117.9 \mathrm{~W}$ & {$[60]$} \\
Jpl & USA & $34.1 \mathrm{~N}$ & $118.1 \mathrm{~W}$ & {$[61]$} \\
Pasadena & USA & $34.1 \mathrm{~N}$ & $118.1 \mathrm{~W}$ & {$[62]$} \\
Zugspitze & Germany & $47.4 \mathrm{~N}$ & $11.0 \mathrm{E}$ & {$[63]$} \\
\hline
\end{tabular}

The authors would like to apologize for any inconvenience caused to the readers by these changes. We would like to add Mr. Xingying Zhang, Dr. Yangmeng Bi, Dr. Ping Wang into the authorship. We would also like to ensure we acknowledge the TCCON partners and their funding agencies using the following text:

Acknowledgments: We thank Minzheng Duan for providing the flight experiment data used in this study. We are also thankful to Zhongdong Yang for providing the Level 1B datasets of TanSat. The TCCON data were obtained from the TCCON Data Archive hosted by CaltechDATA at https: //tccondata.org. We thank TCCON PIs for the TCCON measurements at stations of Bialystok, Bremen, Burgos, Darwin, Edwards, Garmisch, Izana, Jpl, Karlsruhe, Lamont, Orleans, Paris, Park Falls, Pasadena, Rikubetsu, Saga, Sodankyla, Tsukuba, Wollongong, and Zugspitze. The Paris TCCON site has received funding from Sorbonne Université, the French research center CNRS, the French space agency CNES, and Région Île-de-France. The TCCON stations at Rikubetsu, and Burgos are supported in part by the GOSAT series project. Local support for Burgos is provided by the Energy Development Corporation (EDC, Philippines). Darwin and Wollongong TCCON stations are supported by ARC grants DP160100598, LE0668470, DP140101552, DP110103118 and DP0879468. We thank OCO-2 team for the Level 2 XCO2 products used in the comparison with XCO2 retrievals from TanSat. We also thank the European Monitoring of Atmospheric Composition Change/CAMS projects for providing $\mathrm{CO} 2$ model fields and the National Oceanic and Atmospheric Administration (NOAA) Earth System Research Laboratory (ESRL) for providing the CT data. We thank 4 anonymous reviewers for their advices on the improvement of the manuscript.

We also would like to add the following references in our paper and change orginal reference 44 to 64 .

44. Kivi, R.; Heikkinen, P.; Kyrö, E. TCCON data from Sodankylä (FI), Release GGG2014.R0, TCCON Data Archive, hosted by CaltechDATA. Available online: https://doi.org/10.14291/tccon.ggg2014. sodankyla01.R0/1149280 (accessed on 28 May 2020). 
45. Deutscher, N.M.; Notholt, J.; Messerschmidt, J.; Weinzierl, C.; Warneke, T.; Petri, C.; Grupe, P.; Katrynski, K. TCCON data from Bialystok (PL), Release GGG2014.R1, TCCON Data Archive, hosted by CaltechDATA. Available online: https://doi.org/10.14291/tccon.ggg2014.bialystok01.R1/ 1183984 (accessed on 28 May 2020).

46. Notholt, J.; Petri, C.; Warneke, T.; Deutscher, N.M.; Buschmann, M.; Weinzierl, C.; Macatangay, R.C.; Grupe, P. TCCON data from Bremen (DE), Release GGG2014.R0, TCCON Data Archive, hosted by CaltechDATA. Available online: https://doi.org/10.14291/tccon.ggg2014.bremen01.R0/1149275 (accessed on 28 May 2020).

47. Hase, F.; Blumenstock, T.; Dohe, S.; Gross, J.; Kiel, M. TCCON data from Karlsruhe (DE), Release GGG2014.R1, TCCON Data Archive, hosted by CaltechDATA. Available online: https: //doi.org/10.14291/tccon.ggg2014.karlsruhe01.R1/1182416 (accessed on 28 May 2020).

48. Wennberg, P.O.; Roehl, C.; Wunch, D.; Toon, G.C.; Blavier, J.-F.; Washenfelder, R.; Keppel-Aleks, G.; Allen, N.; Ayers, J. TCCON data from Park Falls (US), Release GGG2014.R0, TCCON Data Archive, hosted by CaltechDATA. Available online: https://doi.org/10.14291/tccon.ggg2014.parkfalls01.R0/ 1149161 (accessed on 28 May 2020).

49. Te, Y.; Jeseck, P.; Janssen, C. TCCON data from Paris (FR), Release GGG2014.R0, TCCON Data Archive, hosted by CaltechDATA. Available online: https://doi.org/10.14291/tccon.ggg2014. paris01.R0/1149279 (accessed on 28 May 2020).

50. Blumenstock, T.; Hase, F.; Schneider, M.; Garcia, O.E.; Sepulveda, E. TCCON data from Izana (ES), Release GGG2014.R0, TCCON Data Archive, hosted by CaltechDATA. Available online: https://doi.org/10.14291/tccon.ggg2014.izana01.R0/1149295 (accessed on 28 May 2020).

51. Warneke, T.; Messerschmidt, J.; Notholt, J.; Weinzierl, C.; Deutscher, N.M.; Petri, C.; Grupe, P.; Vuillemin, C.; Truong, F.; Schmidt, M.; Ramonet, M.; Parmentier, E. TCCON data from Orléans (FR), Release GGG2014.R0, TCCON Data Archive, hosted by CaltechDATA. Available online: https://doi.org/10.14291/tccon.ggg2014.orleans01.R0/1149276 (accessed on 28 May 2020).

52. Sussmann, R.; Rettinger, M. TCCON data from Garmisch (DE), Release GGG2014.R0, TCCON Data Archive, hosted by CaltechDATA. Available online: https://doi.org/10.14291/tccon.ggg2014. garmisch01.R0/1149299 (accessed on 28 May 2020).

53. Morino, I., Yokozeki, N., Matsuzaki, T., and Horikawa, M.: TCCON data from Rikubetsu (JP), Release GGG2014.R1, TCCON Data Archive, hosted by CaltechDATA. Available online: https://doi.org/10.14291/tccon.ggg2014.rikubetsu01.R1/1242265 (accessed on 28 May 2020)

54. Wennberg, P.O.; Wunch, D.; Roehl, C.; Blavier, J.-F.; Toon, G.C.; Allen, N. TCCON data from Lamont (US), Release GGG2014.R1, TCCON Data Archive, hosted by CaltechDATA. Available online: https://doi.org/10.14291/tccon.ggg2014.lamont01.R1/1255070 (accessed on 28 May 2020).

55. Morino, I.; Matsuzaki, T.; Horikawa, M. TCCON data from Tsukuba (JP), 125HR, Release GGG2014.R1, TCCON Data Archive, hosted by CaltechDATA. Available online: https://doi.org/ 10.14291/tccon.ggg2014.tsukuba02.R1/1241486 (accessed on 28 May 2020).

56. Kawakami, S.; Ohyama, H.; Arai, K.; Okumura, H.; Taura, C.; Fukamachi, T.; Sakashita, M. TCCON data from Saga (JP), Release GGG2014.R0, TCCON Data Archive, hosted by CaltechDATA. Available online: https://doi.org/10.14291/tccon.ggg2014.saga01.R0/1149283 (accessed on 28 May 2020).

57. Griffith, D.W.T.; Deutscher, N.M.; Velazco, V.A.; Wennberg, P.O.; Yavin, Y.; Keppel-Aleks, G.; Washenfelder, R.; Toon, G.C.; Blavier, J.-F.; Paton-Walsh, C.; et al. TCCON data from Darwin (AU), Release GGG2014.R0, TCCON Data Archive, hosted by CaltechDATA. Available online: https://doi.org/10.14291/tccon.ggg2014.darwin01.R0/1149290 (accessed on 28 May 2020).

58. Griffith, D.W.T.; Velazco, V.A.; Deutscher, N.M.; Paton-Walsh, C.; Jones, N.B.; Wilson, S.R.; Macatangay, R.C.; Kettlewell, G.C.; Buchholz, R.R.; Riggenbach, M. TCCON data from Wollongong (AU), Release GGG2014.R0, TCCON Data Archive, hosted by CaltechDATA. Available online: https://doi.org/10.14291/tccon.ggg2014.wollongong01.R0/1149291 (accessed on 28 May 2020). 
59. Morino, I.; Velazco, V.A.; Hori, A.; Uchino, O.; Griffith DW, T. TCCON data from Burgos, Ilocos Norte (PH), Release GGG2014.R0, TCCON Data Archive, hosted by CaltechDATA. Available online: https://doi.org/10.14291/tccon.ggg2014.burgos01.R0 (accessed on 28 May 2020).

60. Iraci, L.T.; Podolske, J.; Hillyard, P.W.; Roehl, C.; Wennberg, P.O.; Blavier, J.F.; Allen, N.; Wunch, D.; Osterman, G.; Albertson, R. TCCON data from Edwards (US), Release GGG2014.R1, TCCON Data Archive, hosted by CaltechDATA. Available online: https://doi.org/10.14291/tccon.ggg2014. edwards01.R1/1255068 (accessed on 28 May 2020).

61. Wennberg, P.O.; Roehl, C.M.; Blavier, J.-F.; Wunch, D.; Allen, N.T. TCCON data from Jet Propulsion Laboratory (US), 2011, Release GGG2014.R1, TCCON Data Archive, hosted by CaltechDATA. Available online: https://doi.org/10.14291/tccon.ggg2014.jp102.R1/1330096 (accessed on 28 May 2020).

62. Wennberg, P.O.; Wunch, D.; Roehl, C.M.; Blavier, J.-F.; Toon, G.C.; Allen, N.T. TCCON data from Caltech (US), Release GGG2014.R1, TCCON Data Archive, hosted by CaltechDATA. Available online: https://doi.org/10.14291/tccon.ggg2014.pasadena01.R1/1182415 (accessed on 28 May 2020).

63. Sussmann, R.; Rettinger, M. TCCON data from Zugspitze (DE), Release GGG2014.R1, TCCON Data Archive, hosted by CaltechDATA. Available online: https://doi.org/10.14291/tccon.ggg2014. zugspitze01.R1 (accessed on 28 May 2020).

\section{Reference}

1. Wang, S.; van der A, R.J.; Stammes, P.; Wang, W.; Zhang, P.; Lu, N.; Fang, L. Carbon Dioxide Retrieval from TanSat Observations and Validation with TCCON Measurements. Remote Sens. 2020, 12, 2204. [CrossRef]

Publisher's Note: MDPI stays neutral with regard to jurisdictional claims in published maps and institutional affiliations. 